\title{
LA INSERCIÓN LABORAL DE PERSONAS CON DISCAPACIDAD
}

\section{EMPLOYABILITY OF DISABLED PEOPLE}

Pablo Aguilar Conde ${ }^{1}$ Nieves Yusta Sainz ${ }^{2}$

1. Dr. en Economía. Ldo. en Derecho. Dpto. de Economía y Administración de Empresas, Universidad de Burgos (España). E-mail: paguilar@ubu.es

2. Lda. en Economía. Lda. en Derecho. Dpto. de Economía Aplicada, Universidad de Burgos (España). E-mail: niyusa@ubu.es

\section{Citación sugerida:}

Aguilar Conde, P. y Yusta Sainz, N. (2017). La inserción laboral de las personas con discapacidad. $3 C$ Empresa: investigación y pensamiento crítico, 6(2), 1-19. DOI: <http://dx.doi.org/10.17993/3cemp.2017.060230.1-19/>. 


\section{RESUMEN}

El presente trabajo pretende analizar la inserción laboral real de las personas con discapacidad en España mediante una metodología, fundamentalmente, descriptiva. Para ello, analizaremos las principales variables y tasas relacionadas con dicha inserción, comparándolas con los valores de las mismas para la población no discapacitada.

Los resultados sugieren que las personas con discapacidad no están completamente integradas en el mercado laboral. Presentan tasas de actividad más bajas, tasas de desempleo más elevadas y salarios más bajos que el resto de trabajadores. Por este motivo, consideramos necesario que las empresas, que se consideran socialmente responsables, desarrollen diferentes medidas de discriminación positiva, entre otras las que contribuyan a incrementar el empleo y los salarios de este colectivo. Encuadramos el trabajo dentro de economía social y discapacidad, exponiendo las medidas de política laboral que se están aplicando para lograr la plena integración laboral de este colectivo.

\section{ABSTRACT}

This paper aims to analyze the labor integration in favor of the disabled people community in Spain, through a descriptive methodology. We will analyze the main variables and rates related to disabled people in comparison with the values for the non-disabled people.

The results suggest that disabled people are not completely integrated into the labor market. They have lower activity rates, higher unemployment rates and lower wages than non-disabled people. For this reason, we consider that social responsible companies should implement different measures of positive discrimination, particularly those measures that contribute to increase employment and wages of this group. We include this paper in the field of social economy and disability, and will finish it, exposing the labor policy measures that are being developed to achieve the full labor integration of the disabled people.

\section{PALABRAS CLAVE}

Economía Social, Discapacidad, Responsabilidad Social Corporativa, Inserción laboral, Discriminación Positiva.

\section{KEY WORDS}

Social Economy, Disability, Corporate Social Responsibility, Labor Integration, Positive discrimination. 


\section{INTRODUCCIÓN}

Una de las principales características demográficas del siglo XXI, en España, será el envejecimiento de la población, el aumento del número de personas discapacitadas y el incremento de los gastos públicos asociados a la satisfacción de las necesidades de estos colectivos.

Según el Instituto Nacional de Estadística, en España y en el año 2016, la población de 65 o más años de edad representaba el $18 \%$ del total. En 2050, basándonos en las proyecciones de este organismo, esa proporción prácticamente se duplicará, alcanzando el porcentaje de $35,3 \%$ de la población ${ }^{1}$. Estas proyecciones nos muestran que la representación gráfica de población española por edades evoluciona hacia una pirámide invertida, en la que el fuerte envejecimiento va a ser una característica de la sociedad española que entrañará importantes consecuencias económicas y sociales. Una sociedad envejecida necesita importantes ingresos públicos para financiar las pensiones, los gastos sanitarios, farmacéuticos y otras prestaciones sociales.

Al mismo tiempo, los avances médico sanitarios han provocado el aumento de la esperanza de vida al nacer y la reducción de la mortalidad infantil. Según datos del INE, en 1995, de cada 10.000 nacimientos, fallecían antes del primer año aproximadamente 55 personas. Veinte años más tarde esa cifra se redujo a la mitad, alcanzando en 2015 el valor de 27,12. Esta reducción del número de defunciones infantiles, en muchos casos, ha traído como consecuencia implícita un aumento de las personas que desde el nacimiento padecen algún tipo de discapacidad. Nacidos, que hace unas décadas no sobrevivían, actualmente consiguen evolucionar satisfactoriamente, aunque con limitaciones en la realización de las actividades de la vida diaria. También se ha producido un incremento de las enfermedades crónicas, como las enfermedades cardiovasculares, el cáncer o la diabetes, que se han convertido en la primera causa de incapacidad del mundo. Todas estas razones han contribuido a incrementar el número de personas que presentan algún grado de discapacidad.

Estas dos características demográficas de la España del siglo XXI tienen importantes repercusiones económicas, concretamente nos referimos a las consecuencias previsibles en el aumento del gasto público por estas dos causas previamente señaladas.

La sostenibilidad del sistema de pensiones, en nuestro país, no es un tema nuevo en el debate económico y político. Respecto a los ingresos se ha planteado una diversificación de las fuentes de financiación, complementándose las cotizaciones sociales con impuestos. Respecto a los gastos, el atraso en la edad de jubilación parece una necesidad imperante, como consecuencia de la evolución futura de la pirámide poblacional.

\footnotetext{
${ }^{1}$ INE: “Proyecciones de población 2016 - 2066"

2 INE: "Movimiento Natural de Población: Estadística de Defunciones"
} 
Al igual que las personas en edad avanzada, las personas con discapacidad suponen un importante coste para las finanzas públicas, tanto en términos de reducción de ingresos (deducciones familiares en el Impuesto sobre la Renta de las Personas Físicas y otros impuestos), como de aumento de gastos en prestaciones sociales (dependencia, etc..)

Dejando al margen el tema del aumento del gasto público consecuencia del aumento de los pensionistas, al ser un tema ya tratado desde diferentes ámbitos, nos referiremos al problema asociado a la falta de integración laboral de las personas discapacitadas, en edad activa, que se convierte en una necesidad, tanto para la sociedad, como para las mismas personas con discapacidad. Para la sociedad, porque de esta forma, se contribuye a aumentar los recursos públicos necesarios para sostener las prestaciones del estado del bienestar y a minorar los gastos (pensiones de invalidez, prestaciones de dependencia relacionadas negativamente con la renta del perceptor); y para las personas afectadas, porque la integración laboral contribuye a lograr una mayor independencia económica, convirtiéndose en un medio para conseguir una adecuada integración social.

Así el objetivo fundamental del presente trabajo es analizar en qué medida las personas con discapacidad en España se han integrado en el mercado laboral. Para tal fin, hemos dividido el trabajo en cinco apartados. A continuación de esta introducción, el segundo apartado perfila el marco conceptual que sustenta gran parte de esta comunicación. En el tercer apartado, creemos necesario hacer una breve enumeración del marco legal asociado con la protección de las personas con discapacidad en el ámbito laboral. El cuarto apartado, desarrolla el análisis empírico: el objetivo y metodología, junto a la presentación y análisis de los resultados. Para finalizar, en el quinto apartado, recoge las conclusiones más relevantes de la presente comunicación.

\section{MARCO CONCEPTUAL}

La Organización Mundial de la Salud define la discapacidad como un término general para las deficiencias, las limitaciones de la actividad y las restricciones en la participación. Es la interacción entre los individuos con un problema de salud (ej. Parálisis cerebral, síndrome de Down, etc.) y factores personales y ambientales (ej. Actitudes negativas, transporte inaccesible o apoyos sociales limitados). ${ }^{3}$

En la normativa española, se considera persona con discapacidad "aquella que presenta deficiencias físicas, mentales, intelectuales o sensoriales, previsiblemente permanentes que, al interactuar con diversas barreras, pueden impedir su participación plena y efectiva, en la sociedad, en igualdad de condiciones con los demás" 4

${ }^{3}$ http://www.who.int/mediacentre/factsheets/fs352/en/, (disponible en enero de 2017).

${ }^{4}$ Art. 4.1 del Real Decreto Legislativo 1/2013, de 29 de diciembre, por el que se aprueba el Texto Refundido de la Ley General de derechos de las personas con discapacidad y de su inclusión social. 
A todos los efectos, tienen la consideración de personas con discapacidad:

- Aquellas a quienes se les ha reconocido un grado de discapacidad igual o superior al $33 \%$.

- Los pensionistas de la Seguridad Social que tienen reconocida una pensión de incapacidad permanente en el grado de total, absoluta o gran invalidez.

- Se considera que presentan una discapacidad en grado igual o superior al $33 \%$ los pensionistas de clases pasivas que tengan reconocida una pensión de jubilación o de retiro por incapacidad permanente para el servicio o inutilidad. ${ }^{5}$

Según la base de datos de personas con discapacidad, a 1 de enero de 2015, las personas discapacitadas representaban un 6,03\% de la población total española (2.813.592 personas con alguna discapacidad; de las cuales 1.410 .310 son mujeres $(50,12 \%)$ y 1.403 .282 son hombres, 49,88\%). Este porcentaje desciende al 5,09\% si solo consideramos las personas con edades comprendidas entre 18 y 64 años $^{6}$ (el número de personas discapacitadas se reduce a 1.488.469). Estos porcentajes son muy similares al porcentaje de personas que reconocen importantes limitaciones en las actividades de la vida diaria.

En el año 2015, a nivel nacional un 5,1\% de las personas de 16 o más años declaraba estar gravemente limitado en las actividades diarias, en los últimos 6 meses por problemas de salud $^{7}$. Este porcentaje es superior para las mujeres $(5,8 \%)$ que para los hombres $(4,4 \%)$. El porcentaje de personas, de 16 años o más, que manifiestan estar gravemente limitado ha disminuido en los últimos 10 años; el decrecimiento más drástico coincidió en el año $2008^{8}$, año de comienzo de la recesión en España. En la misma medida, se ha incrementado el porcentaje de personas que declaran tener limitaciones, aunque no graves, en las actividades de la vida diaria ${ }^{9}$. Los datos nos sugieren que la mayor parte de este colectivo (personas que tienen limitaciones pero no graves) no tiene reconocido grado de discapacidad, y por tanto, no es objeto de nuestro trabajo.

\footnotetext{
${ }^{5}$ Art. 4.2. Real Decreto Legislativo 1/2013.

${ }^{6}$ Elegimos este tramo de edad, porque los datos que nos proporciona la Base de Datos Estatal de Personas con Discapacidad se corresponden a este tramo de edad.

${ }^{7}$ Encuesta de calidad de vida, INE.

${ }^{8}$ En 2007, el porcentaje de personas de 16 o más años que declaraba estar gravemente limitado en las actividades de la vida diaria era de un 9,1\%; este porcentaje descendió hasta el 5,4\% en el año 2008.

${ }^{9}$ En 2007, el porcentaje de personas de 16 o más años que declaraban tener limitaciones en la vida diaria, aunque no graves, era del $14,4 \%$; un año más tarde se situaba en el $17,3 \%$; alcanzando el máximo en 2015 con un valor del 19,6\%.
} 


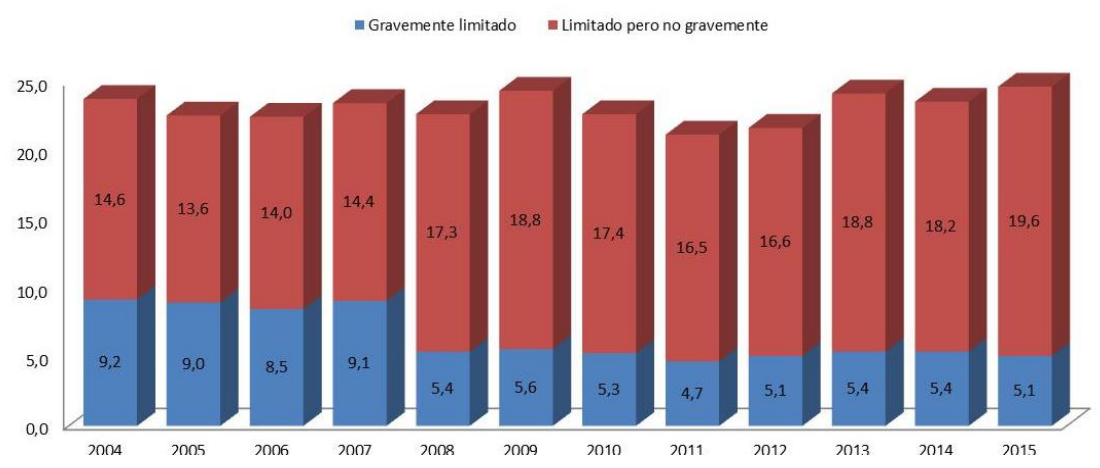

Gráfico 1. Personas con limitaciones en la actividad diaria (2015). \% de personas de 16 o más años.

Fuente: Elaboración propia a partir de los datos de la Encuesta de Calidad de Vida.

Como es de suponer, el porcentaje de personas que declaran estar gravemente limitadas, o estar limitadas pero no gravemente, se incrementa al aumentar la edad. Sin embargo, disminuye al elevarse el nivel de educación.

Según el Eurostat, en la UE, en 2015, el 25,3\% de las personas de 16 o más años, consideraba que presentaba limitaciones a largo plazo en las actividades de la vida diaria, debido a problemas de salud. Este porcentaje es superior para las mujeres $27,5 \%$, que para los hombres (23\%). También existe una diferencia según el nivel de renta. Entre las personas más pobres, el 31,2\% presenta dificultades en las actividades de la vida diaria, mientras que, entre los más ricos, solo el $17 \%$ se enfrenta a este problema. Los países que tienen un menor porcentaje de personas que consideran que tienen limitaciones a largo plazo en las actividades habituales son Malta, 9,7\%, y Suecia, 11,1\%. En cambio, los que tienen un porcentaje mayor son Letonia, $38,5 \%$ y Portugal, $36,1 \%^{10}$.

A nivel mundial, la Organización Mundial de la Salud estimó, en 2011, que el 15\% de la población mundial tenía alguna forma de discapacidad ${ }^{11}$.

Por tanto, a partir de los datos existentes, existe una diferencia entre el número de personas reconocidas a todos los efectos como discapacitadas y el número de personas que declaran tener ciertas limitaciones en la realización de las actividades de la vida diaria. El reconocimiento de un cierto grado de discapacidad está vinculado a graves limitaciones en la vida diaria que dificultarán la participación en el mercado de trabajo.

Según la encuesta "El empleo de las personas con discapacidad", en el año 2015 aproximadamente el $11 \%$ de los hogares que tienen alguna persona de 16-64, cuentan con algún miembro con discapacidad. Sin embargo, muchas personas discapacitadas residen en hogares colectivos, por lo que no se contabilizarían en la encuesta anterior.

10 http://ec.europa.eu/eurostat/documents/2995521/7755879/3-02122016-BP-EN.pdf/a4d93b5a78f3-46df-8cec-eb83f48ab3a7

${ }^{11}$ http://www.who.int/mediacentre/factsheets/fs352/en/ 


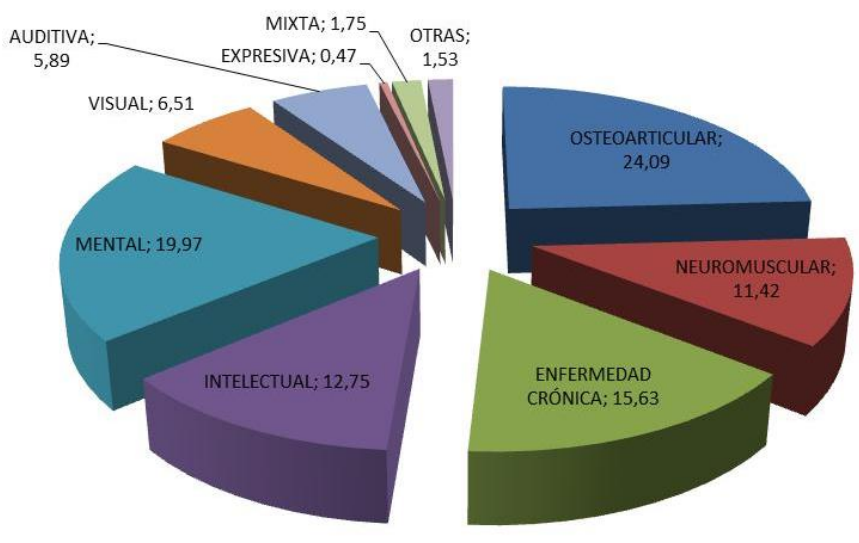

Gráfico 2. Distribución porcentual de las personas con grado de discapacidad reconocido igual o superior al 33\% según tipo de primera deficiencia (entre 18 y 64 años). 2015.

Fuente: Base Estatal de datos de Personas con Discapacidad.

Como podemos comprobar en el gráfico precedente, casi una cuarta parte de las personas con discapacidad de entre 18 y 64 años, presentan problemas osteo articulares, lo que dificultará el desempeño de empleos que supongan una sobrecarga física, pero no impide la realización de tareas donde se exija un esfuerzo más intelectual u otro tipo de habilidades personales. La quinta parte de las personas discapacitadas tienen como primera deficiencia una enfermedad mental. Las enfermedades mentales incluyen desde las demencias, esquizofrenias hasta los trastornos de la conducta alimentaria (como la anorexia o bulimia).

El grado de discapacidad nos muestra las dificultades que un individuo tiene para realizar actividades. Por tanto, cuanto mayor sea el grado, mayor serán las limitaciones para realizar actividades.

Respecto al grado de discapacidad, podemos observar en el grafico siguiente, que prácticamente el $60 \%$ de las personas con discapacidad, tienen un grado moderado de la misma (entre el $33 \%$ y $64 \%$ ). Estas personas, con las adaptaciones necesarias pueden desempeñar un puesto de trabajo. Los grados superiores de discapacidad exigen mayores adaptaciones y, en algunos casos, empleos en centros especiales. 


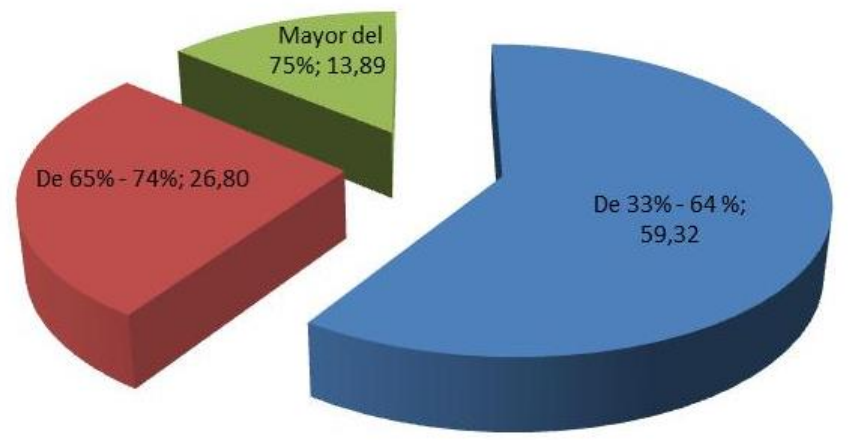

Gráfico 3. Distribución porcentual de las personas con discapacidad reconocida igual o superior al 33\% por grado de discapacidad (entre 18 y 64 años). Año 2015.

Fuente: Base Estatal de Datos de Personas con Discapacidad.

\section{MARCO LEGAL}

El Art. 37 del Real Decreto Legislativo 1/ 2013, de 29 de noviembre, por el que se aprueba el texto refundido de la Ley General de derechos de las personas con discapacidad y su inclusión social, establece que las personas con discapacidad pueden ejercer su derecho al trabajo a través de los siguientes tipos de empleo:

a. Empleo ordinario en las empresas y en las administraciones públicas, incluidos los servicios de empleo con apoyo.

b. Empleo protegido, en Centros Especiales de Empleo y a través de Enclaves Laborales.

c. Empleo autónomo.

Resumiremos las principales medidas de política activa de empleo que se han adoptado para fomentar la contratación del colectivo analizado tanto en el empleo ordinario como en el empleo protegido.

\section{a. EMPLEO ORDINARIO}

1. Cuota de reserva: Todas aquellas empresas públicas y privadas que empleen un número de trabajadores superior a 50, están obligadas a emplear como mínimo un $2 \%$ de trabajadores con discapacidad(Art. 42, RD 1/2013) $)^{12}$, salvo convenio

\footnotetext{
${ }^{12}$ El Art. 1 del Real Decreto 364/2005, de 8 de abril, por el que se regula el cumplimiento alternativo con carácter excepcional de la cuota de reserva a favor de los trabajadores con discapacidad menciona dos excepciones para esta obligación general:

- Imposibilidad de que los Servicios Públicos de Empleo o las Agencias de Colocación puedan atender la oferta de empleo después de haber efectuado la gestiones de intermediación necesarias.

- Acreditación por parte de la empresa de cuestiones de carácter productivo, organizativo, técnico o económico que motiven especial dificultad para incorporar trabajadores con discapacidad.
} 
colectivo o voluntad del empresario en cuyo caso se aplicarán medidas alternativas ${ }^{13}$.

En las ofertas de empleo público, la Administración tendrá que reservar un $7 \%$ de las vacantes a personas con discapacidad ${ }^{14}$.

\section{Contratación por cuenta ajena:}

Coexisten diversas ayudas e incentivos a la contratación de personas con discapacidad, que dependerán del tipo de contrato y del grado de discapacidad:

a. Contratación indefinida. Las ayudas que nos encontramos a la contratación de este colectivo, a través del contrato indefinido son:

Por un lado, subvenciones directas, 3.907€ si el trabajador discapacitado es contratado a jornada completa; si es contratado a tiempo parcial, la cantidad anterior se reducirá proporcionalmente en función de la jornada establecida ${ }^{15}$.

Adicionalmente, bonificaciones de las cuotas de la Seguridad Social durante la vigencia del contrato que dependen de la edad y del grado de discapacidad, severa o no severa ${ }^{16}$.

Tabla 1. Bonificaciones de las cuotas de la seguridad social durante la vigencia del contrato. ${ }^{17}$

\begin{tabular}{|c|c|c|c|c|}
\hline & \multicolumn{2}{|c|}{ SIN DISCAPACIDAD SEVERA } & \multicolumn{2}{c|}{ CON DISCAPACIDAD SEVERA } \\
\hline $\begin{array}{c}\text { MENORES DE } \\
45 \text { AÑOS }\end{array}$ & HOMBRES & MUJERES & HOMBRES & MUJERES \\
\hline $\begin{array}{c}4.500 € \text { año } \\
\text { AÑNOS }\end{array}$ & $\begin{array}{c}5.350 € \text { año } \\
\text { HOMBRES }\end{array}$ & MUJERES & HOMBRES & MUJERES \\
\hline & $5.700 €$ año & $5.700 €$ año & $6.300 €$ año & $6.300 €$ año \\
\hline
\end{tabular}

Fuente: elaboración propia.

${ }^{13}$ Las medidas alternativas están recogidas en el Art. 2, RD 364/2005:

- Realización de un contrato mercantil o civil con un Centro Especial de Empleo o con un trabajador autónomo con discapacidad (para el suministro de bienes, o para la prestación de servicios ajenos)

- Realización de donaciones o acciones de patrocinio.

- Constitución de Enclaves Laborales.

${ }^{14}$ Art. 59 del Real Decreto legislativo, de 30 de octubre, por el que se aprueba el texto refundido de la Ley del Estatuto Básico del Empleado Público.

${ }^{15}$ Art. 7 del Real Decreto 1451/1983 de 11 de mayo, por el que en cumplimiento de lo previsto en la ley 13/1982, de 7 de abril, se regula el empleo selectivo y las medidas de fomento del empleo de los trabajadores discapacitados; en la redacción dada por el artículo uno del Real Decreto 170/ 2004, de 30 de enero, de modificación del anterior.

${ }^{16}$ Se considera que sufren discapacidad severa las personas con parálisis cerebral, con enfermedad mental o con discapacidad intelectual, con un grado de discapacidad reconocido igual o superior al $33 \%$ y las personas con discapacidad física o sensorial, con un grado de discapacidad reconocido igual o superior al $65 \%$.

${ }^{17}$ Art. 2.2 Ley 43/2006, de 29 de diciembre, para la mejora del crecimiento y del empleo. 
Además de los beneficios anteriores, se mantienen deducciones de la cuota íntegra del impuesto sobre sociedades:

- 9.000€ por persona y año en que se haya incrementado el promedio de trabajadores en plantilla con un grado de discapacidad igual o superior al $33 \%$.

- $\quad 12.000 €$ persona y año en que se haya incrementado el promedio de trabajadores en plantilla con un grado de discapacidad igual o superior al $65 \%{ }^{18}$.

b. Contrato temporal de fomento del empleo. El art. 2, Ley 43/2006, establece las bonificaciones de las cuotas de la Seguridad Social, a las que tendrán derecho los empleadores que contraten a personas con discapacidad a través de esta fórmula contractual.

Tabla 2. Bonificaciones de las cuotas de la seguridad social durante la viegencia del contrato.

\begin{tabular}{|l|l|l|l|l|}
\hline & \multicolumn{2}{|l|}{ SIN DISCAPACIDAD SEVERA } & \multicolumn{2}{l|}{ CON DISCAPACIDAD SEVERA } \\
\hline $\begin{array}{l}\text { MENORES DE 45 } \\
\text { AÑOS }\end{array}$ & HOMBRES & MUJERES & HOMBRES & MUJERES \\
\hline & $3.500 €$ año & $4.100 €$ año & $4.100 €$ año & $4.700 €$ año \\
\hline 45 O MÁS AÑOS & HOMBRES & MUJERES & HOMBRES & MUJERES \\
\hline & $4.100 €$ año & $4.700 €$ año & $4.700 €$ año & $5.300 €$ año \\
\hline
\end{tabular}

Fuente: elaboración propia.

Adicionalmente a las ayudas y las bonificaciones anteriores, las empresas contratantes pueden optar a las subvenciones destinadas a financiar los costes laborales y de Seguridad Social generados por los preparadores laborales especializados, contratados para prestar servicios de orientación y acompañamiento individualizado a los trabajadores con discapacidad con especiales dificultades de inserción laboral, con el objetivo de facilitar su adaptación laboral.

La cuantía de estas ayudas depende del tipo y grado de discapacidad ${ }^{19}$ :

- Cuando el trabajador sufra parálisis cerebral, enfermedad mental o discapacidad intelectual, la subvención será de $4000 €$ al año si el grado de discapacidad es igual o superior al 33\% e inferior al 65\%; y de 6.600 euros al año si el grado de discapacidad es igual o superior al $65 \%$.

- Si el trabajador tiene una discapacidad física o sensorial con un grado de discapacidad reconocido igual o superior al $65 \%$ y en el caso de personas sordas y con discapacidad auditiva con un grado de discapacidad reconocido igual o superior al 33\%, la cuantía de la subvención será de 2.500 euros año.

\footnotetext{
${ }^{18}$ Art. 38, Ley 27/2014, de 27 de noviembre, del Impuesto sobre Sociedades.

${ }^{19}$ Art. 8, Real Decreto 870/2007, de 2 de julio, por el que se regula el programa de empleo con apoyo como medida de fomento del empleo de personas con discapacidad en el mercado ordinario de trabajo.
} 


\section{b. EMPLEO PROTEGIDO}

La integración laboral de las personas con discapacidad en el sistema protegido de trabajo se va a llevar a cabo en los Centros Especiales de Empleo y a través de enclaves laborales ${ }^{20}$. El objetivo principal de los Centros Especiales de Empleo es realizar una actividad productiva de bienes y servicios asegurando un empleo remunerado a las personas con discapacidad (al menos un $70 \%$ de la plantilla ha de estar formada por personas con discapacidad) $)^{21}$.

Para promocionar la integración de los trabajadores discapacitados en los centros especiales de empleo, se establecen las siguientes ayudas ${ }^{22}$ :

- Subvenciones para financiar proyectos generadores de empleo. Por cada puesto de trabajo estable creado el centro recibirá 9.015,18 euros, si el número de trabajadores con discapacidad en plantilla es menor del 90\%; o 12.020,24, si el anterior porcentaje es superior al $90 \%$. Estas subvenciones estarán destinadas a financiar:

$>$ Asistencia técnica (estudios de viabilidad, auditorías, informes económicos y asesoramiento en las diversas áreas de gestión empresarial).

$>$ Intereses de los préstamos contraídos con entidades financieras que tengan subscrito el convenio con el Ministerio de Empleo (hasta un máximo de tres puntos sobre el tipo de interés contratado).

$>$ Inversión fija en proyectos de reconocido interés social.

- Ayudas para apoyo al mantenimiento de los puestos de trabajo:

$>$ Bonificación del $100 \%$ de la cuota empresarial por todas las contingencias a la Seguridad Social por contrato indefinido y temporal.

$>$ Subvención del coste salarial (hasta un máximo del 50\% del salario mínimo interprofesional)

> Subvenciones para adaptación del puesto y eliminación de barreras (un máximo de $1.803 €$ por puesto de trabajo creado).

Subvención para equilibrar y sanear financieramente los Centros Especiales de Empleo.

$>$ Subvención destinada a equilibrar el presupuesto de los Centros Especiales que no tengan ánimo de lucro o hayan sido declarados de utilidad pública.

$>$ Asistencia técnica.

\footnotetext{
20 Los enclaves laborales son contratos entre las empresas del mercado ordinario de trabajo y los centros especiales de empleo para la realización de obras o servicios por los que un grupo de trabajadores con discapacidad del centro especial de empleo se desplazan temporalmente al centro de trabajo de la empresa del mercado ordinario.

${ }^{21}$ Art. 43, Real Decreto Legislativo 1/2013.

22 Art. 4, Orden 16 de octubre de 1998 por la que se establecen las bases reguladoras para la concesión de ayudas y subvenciones públicas destinadas al fomento de la integración laboral de los discapacitados en centros especiales de empleo y trabajo autónomo.
} 


\section{ANÁLISIS EMPÍRICO}

\subsection{OBJETIVO Y METODOLOGÍA}

Tal y como avanzamos en la introducción, el objetivo principal de la presente comunicación es determinar el grado de integración en el mercado laboral español de las personas con discapacidad.

Para tal fin, desarrollaremos un análisis fundamentalmente descriptivo, acudiendo a las diferentes fuentes de datos señaladas a continuación.

Inicialmente, como hemos señalado en el marco conceptual, hemos utilizado la Base Estatal de Datos de Personas con Discapacidad, publicada por el Instituto de Mayores y Servicios Sociales (IMSERSO). Esta fuente estadística nos ofrece datos anuales, desde el 31 de diciembre de 2011, hasta el último día de 2014. Cuando nos referimos al número de discapacitados a 1 de enero de 2015, estamos utilizando el informe del último día de 2014.

Respecto a las tasas de participación en el mercado de trabajo hemos utilizado la encuesta publicada por EI INE, "El empleo de las personas con discapacidad". Operación estadística que tiene como objetivo obtener datos de la oferta de trabajo (ocupados y parados) y de las personas ajenas al mercado laboral (inactivos) del colectivo de personas con discapacidad oficialmente reconocida, combinando la información obtenida de la Encuesta de Población Activa (los 4 trimestres del año de referencia) con la Base de Datos Estatal de Personas con discapacidad (a 31 de diciembre del año anterior al de referencia).

El ámbito poblacional de esta encuesta será las personas de $16-64$ años con un grado de discapacidad mayor o igual a $33 \%$ y asimilados, que residen en viviendas familiares principales.

A la hora de analizar los datos de actividad, tenemos que hacer una matización. Si utilizamos la Encuesta de Población Activa (EPA) observamos que las tasas de actividad de la población española son menores que las tasas de actividad que nos proporciona "El empleo de las personas con discapacidad". Esto es debido a dos razones: La primera se refiere al diferente cálculo de la misma por estas dos fuentes. Por un lado, la EPA calcula la tasa de actividad como el cociente entre el número de activos y las personas de 16 años o más; en cambio, "El empleo de las personas con discapacidad", a la hora de calcular la tasa de actividad, lo realiza dividiendo el número de activos entre la población de 16 a 64 años. Al ser menor el denominador, en esta última encuesta, las tasas de actividad serán mayores que las proporcionadas por la EPA. La EPA nos proporciona las tasas de actividad para ambos colectivos, no distinguiendo entre personas con discapacidad y personas sin discapacidad, lo que da lugar a que la tasa global sea menor que la tasa de actividad de las personas sin discapacidad. 
Para analizar los datos sobre diferencias salariales hemos utilizado "El salario de las personas con discapacidad" que es una operación publicada por el INE que tiene como finalidad comparar el salario de los trabajadores por cuenta ajena sin discapacidad y los que tienen reconocido un cierto grado de discapacidad. Para ello integran los datos de la "Encuesta de Estructura Salarial" con los de la "Base de Datos Estatal de Personas con discapacidad" y los datos de la Tesorería General de la Seguridad Social sobre fomento del empleo. El problema al que nos enfrentamos es que los últimos datos disponibles corresponden a 2014 (publicados en febrero de 2017).

\subsection{PRESENTACIÓN Y ANÁLISIS DE LOS RESULTADOS}

Como es predecible, existe una importante diferencia entre las tasas de actividad entre las personas con discapacidad y las personas sin discapacidad (en el caso de las mujeres esta diferencia es de 40 puntos porcentuales, y en el caso de los varones es de 50 puntos porcentuales); las segundas tienen tasas de participación que doblan las del colectivo analizado. Las razones para ello pueden ser de diversa índole: Mayores dificultades de acceso al mercado de trabajo relacionadas con las actitudes de los empleados, menor número de empleos u ocupaciones que pueden desempeñar o que poseen las adaptaciones necesarias y existencia de rentas no laborales superiores al salario de reserva que desincentivan el incorporarse a la población activa

Muchas personas con discapacidad suelen tener reconocida una pensión de incapacidad o pensión no contributiva $y$, como nos explica la teoría económica ${ }^{23}$, la presencia de rentas no laborales genera un efecto renta que provoca una disminución de la participación en el mercado de trabajo.

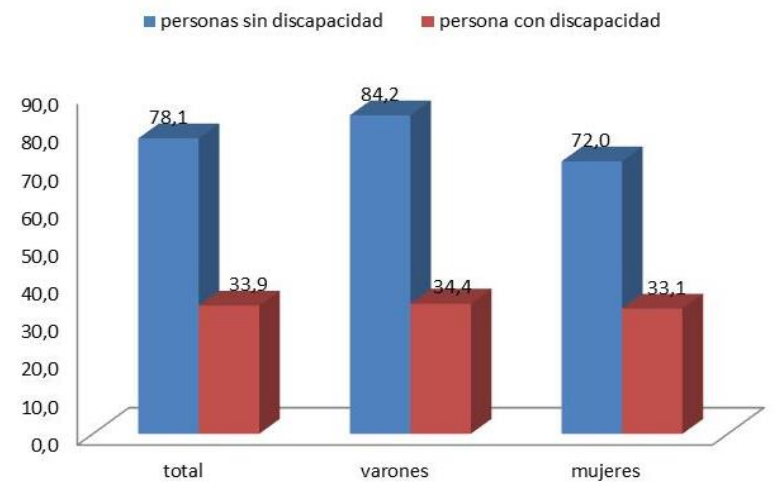

Gráfico 4. Tasa de actividad 2015.

Fuente: El empleo de las personas con discapacidad. INE.

\footnotetext{
23 La existencia de rentas no laborales produce un efecto renta que induce al individuo a desear disfrutar de más ocio, a costa de trabajar menos horas.
} 
Otro motivo para esta menor tasa de actividad puede ser la no búsqueda de empleo porque creen que no lo van a encontrar. En épocas en las que el empleo es insuficiente aumentan las dificultades para que las personas con discapacidad encuentren un empleo adecuado a sus características.

Si dividimos la población en 3 grupos de edad: de $16-24$ años, de 25 a 44 años, y de 45 a 64 años, observamos lo siguiente:

- Las personas con discapacidad tienen bajas tasas de actividad en las edades jóvenes (lo mismo ocurre con las personas que no sufren discapacidad), quizá porque estén todavía en proceso formativo.

- Aumenta la tasa de actividad en el grupo de edad media, de 25 a 44 años. Sin embargo, la diferencia entre las tasas de actividad de las personas discapacitadas y las que no lo son es de 42 puntos porcentuales, a favor de las segundas.

- Pero, donde se alcanza una mayor diferencia en las tasas de actividad, es en el grupo de edad próximo a la jubilación, de 45 - 64 años; siendo la diferencia de 48 puntos porcentuales.

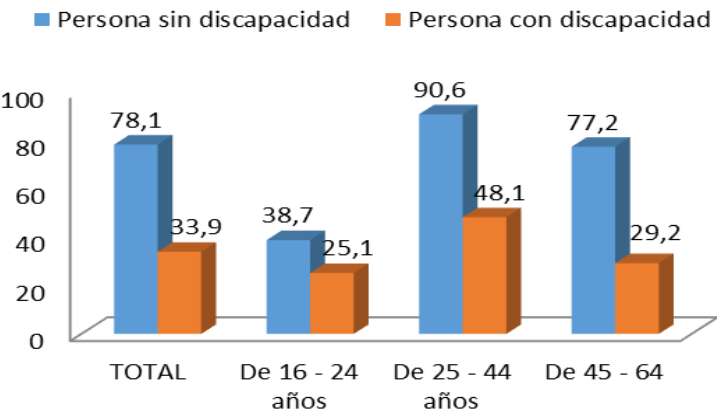

Gráfico 5. Tasa de actividad por grupo de edad (2015).

Fuente: Elaboración propia a partir de los datos de "El empleo de las personas con discapacidad". INE.

La diferencia entre las tasas de actividad de ambos colectivos, aun siendo elevada, es menor, en los municipios más grandes; es decir, disminuye con el tamaño del municipio. Las oportunidades de empleo para todos los colectivos, y en especial para las personas con discapacidad, son mayores en municipios más grandes debido a que en éstos existe una mayor variedad de puestos de trabajo que exigen cualificaciones diferentes $y$, por tanto, acordes con las capacidades de este colectivo. Las empresas son más grandes y con más empleados lo que abarata la introducción de las adaptaciones necesarias; además, el cumplimiento de la cuota de reserva ${ }^{24}$, en las empresas más grandes, genera un aumento de las oportunidades de empleo para el colectivo analizado.

\footnotetext{
${ }^{24}$ La cuota de reserva es la obligación que tienen las empresas españolas con más de 50 trabajadores de emplear como mínimo un $2 \%$ de trabajadores con discapacidad excepto que se apliquen medidas alternativas como son la realización de un contrato mercantil o civil con un Centro Especial de
} 
Esto provoca, que en las áreas más dispersas la diferencia entre las tasas de actividad sea mayor que en las áreas más densamente pobladas.

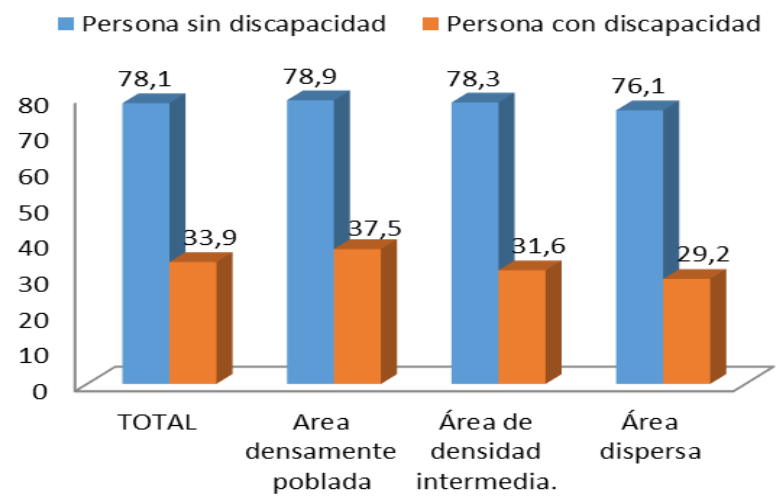

Gráfico 6. Tasa de actividad según el tamaño del municipio (2015).

Fuente: Elaboración propia a partir de los datos de "El empleo de las personas con discapacidad".

INE.

Cuando nos centramos en las personas que están disponibles para trabajar, observamos que la tasa de paro es mayor también para las personas con discapacidad, que tienen prácticamente la misma tasa de paro por sexos y que soportan tasas entre 8 o 10 puntos porcentuales superiores a las personas sin discapacidad. El motivo fundamental, será la mayor dificultad que tienen estas personas, a ser contratadas por parte de los empleadores.

Las empresas, inicialmente, pueden ser más reticentes a la contratación de estas personas por sus dudas sobre la productividad de las mismas (existe la creencia que las personas con discapacidad son menos productivas, debido a sus limitaciones, que el resto de trabajadores, en el seno de la empresa) o su formación. Sin embargo, existen otros factores que pueden incentivar su contratación como puede ser el menor coste económico que suponen y la sensibilidad y empatía hacia este colectivo. Además, las personas con discapacidad pueden desarrollar otras capacidades o habilidades, como puede ser la responsabilidad, motivación, iniciativa, paciencia, necesarias para el desempeño de muchos empleos.

El diferencia entre la tasa de paro entre las personas con discapacidad y las personas sin discapacidad disminuye conforme aumenta la edad. Los jóvenes de 16 a 24 años con discapacidad sufren tasas de paro superiores en 15,3 puntos porcentuales respecto a sus coetáneos sin discapacidad. Esta diferencia se reduce a 12,9 puntos para las personas de 25 a 44 años y a 8,6 puntos para los trabajadores de 45 a 64 años.

Empleo o con un trabajador autónomo con discapacidad, la realización de donaciones o acciones de patrocinio, o la constitución de enclaves laborales. 


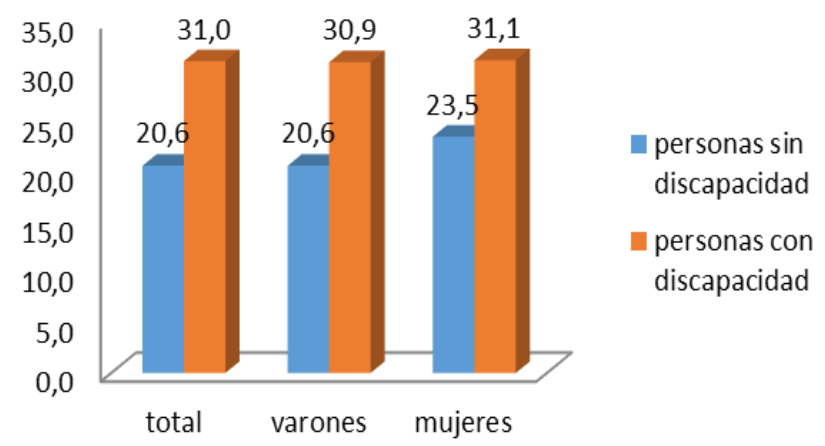

Gráfico 7. Tasa de paro 2015.

Fuente: Elaboración propia a partir de los datos de "El empleo de las personas con discapacidad". INE.

Respecto a los salarios, existe también una importante diferencia entre las ganancias brutas por trabajador y año, entre personas con discapacidad y personas sin discapacidad. Además, esta diferencia ha aumentado desde 2010 hasta 2014 (últimos datos disponibles).

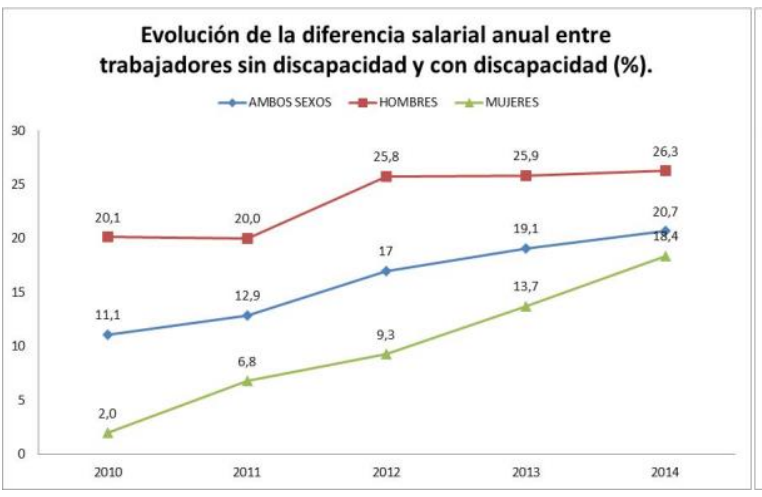

Gráfico 8. Evolución de la diferencia salarial anual entre trabajadores sin discapacidad y con discapacidad (\%).

Fuente: elaboración propia.

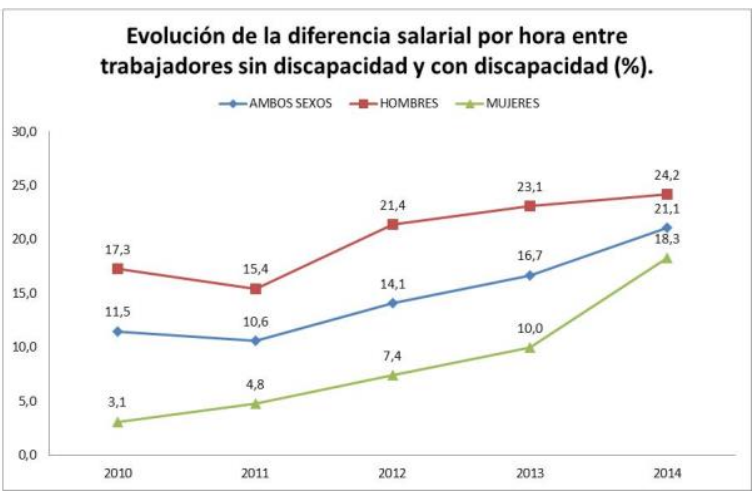

Gráfico 9. Evolución de la diferencia salarial por hora entre trabajadores sin discapacidad y con discapacidad (\%).

Fuente: elaboración propia.

En el año 2010, las personas sin discapacidad ganaban, en términos anuales, un 11,1\% más que las personas con discapacidad. En el año 2014, esta diferencia era del 20,7\%. Esta diferencia es mayor en los varones que en las mujeres. La razón puede ser, que al ser mayores las ganancias medias de los hombres sin discapacidad (existiendo una importante dispersión respecto a la media), las diferencias en las ganancias medias brutas anuales serán mayores. Sin embargo, desde el comienzo del periodo analizado la diferencia salarial ha aumentado exponencialmente para el colectivo femenino. 
Se podría pensar que uno de los motivos por los que las personas sin discapacidad obtienen una mayor ganancia anual es porque realizan más horas. Si comparamos la diferencia porcentual bruta por hora, la disparidad es menor, para el caso de los varones; pero no ocurre esto para las mujeres. En el caso del colectivo femenino, observamos que prácticamente la diferencia salarial entre ambos grupos (trabajadoras sin discapacidad y trabajadoras con discapacidad) es la misma si comparamos la ganancia anual y la ganancia por hora; incluso, algunos años, es mayor la diferencia salarial bruta por hora, lo que nos puede sugerir que, en esos periodos las mujeres discapacitadas han realizado más horas que sus homólogas sin discapacidad.

\section{CONCLUSIONES}

En resumen, consideramos que no se ha producido una plena integración en el mercado laboral de las personas con discapacidad, por las siguientes razones, entre otras:

Sobre participación en el mercado de trabajo: Las personas con discapacidad soportan menores tasas de actividad que las personas sin discapacidad. La diferencia entre ambas tasas aumenta conforme se incrementa la edad y se reduce cuanto mayor es el municipio de residencia.

Sobre desempleo: Los trabajadores con discapacidad sufren tasas de paro superiores alrededor de 9 puntos porcentuales respecto a sus homólogos sin discapacidad. Esto es debido, a pesar de la aplicación de importantes medidas de políticas activas, a la mayor dificultad de colocación y contratación que padece el colectivo.

Sobre salario: Las personas sin discapacidad disfrutan, aproximadamente, de salarios más elevados que las personas con discapacidad. Esta diferencia, que oscila alrededor del $20 \%$, se acentúa en el caso de los varones.

Respecto a las perspectivas futuras, consideramos que la discapacidad aumentará en el futuro. Como la discapacidad aumenta con la edad, el aumento en la esperanza de vida dará lugar a un incremento del porcentaje de personas con discapacidad; también se incrementará debido a la aparición de nuevas enfermedades raras, de carácter crónico, en su mayoría genéticas pero también vinculadas a la exposición ambiental. El atraso en la edad de jubilación, la mayor tasa de supervivencia en el nacimiento y de los accidentes de tráfico, provocarán, así mismo, un incremento considerable del porcentaje de personas discapacitadas.

No todas las deficiencias que llevan aparejada una discapacidad implican una incapacidad para desempeñar una prestación laboral. Una discapacidad intelectual es perfectamente compatible con un empleo que exija capacidades o habilidades físicas y una discapacidad física puede ser compatible con el desempeño de un empleo que exija un esfuerzo intelectual. 
La incorporación del colectivo de personas discapacitadas al mercado laboral dependerá, entre otros factores, del tipo de enfermedad o limitaciones que padezca, del grado de dichas limitaciones, de la efectividad de los medicamentos o ayudas técnicas para paliar los efectos de dichas limitaciones, de las adaptaciones existentes en el entorno de trabajo y de la existencia de incentivos fiscales o subvenciones que contribuyan a reducir los costes de las adaptaciones necesarias. Muchos países han empezado a adoptar medidas para una mayor inserción de las personas con discapacidad; sin embargo, a pesar de eso, no existe una completa integración de las personas con discapacidad en el mercado de trabajo.

Entre las medidas que se han aplicado para lograr el objetivo anterior podemos encontrar subvenciones directas a la contratación indefinida, bonificaciones en las cuotas de la Seguridad Social durante la vigencia del contrato y deducciones en la cuota íntegra del impuesto sobre sociedades. Destaca también, en el empleo ordinario, la cuota de reserva u obligación que tienen las empresas con más de 50 trabajadores de emplear como mínimo un $2 \%$ de trabajadores con discapacidad. En el empleo protegido generado por los centros especiales de empleo existen también subvenciones del coste salarial o para equilibrar o sanear financieramente el centro especial de empleo.

Creemos que con este trabajo se ha ampliado el estudio descriptivo asociado con la integración laboral de las personas con discapacidad. No obstante, a pesar de estas humildes aportaciones, debemos reconocer en este punto las carencias y limitaciones de este trabajo, de tal forma que sirva de incentivo para continuar esta línea de investigación.

Así, para conocer la eficacia de las medidas de política socio laboral destinadas a este grupo es necesario disponer de datos que nos permitan conocer la situación real de las personas con discapacidad en el mercado de trabajo; ya que, la ausencia de datos puede dificultar su comprensión e impedir que se adopten medidas oportunas. A la hora de realizar el trabajo nos hemos encontrado con la importante limitación que supone la escasez de datos actualizados. Así por ejemplo, los datos de "Los salarios de las personas con discapacidad" corresponden a 2014 (publicados en febrero de 2017).

Todas las limitaciones creemos que pueden justificar las posibilidades de ampliación de este trabajo, que lo consideramos como un estímulo para seguir cubriendo etapas en el estudio de la integración laboral de las personas con discapacidad.

Dentro de las líneas futuras de investigación, apuntamos las siguientes:

- Análisis de los determinantes que explican las diferencias en las tasas de paro y salarios de los trabajadores con discapacidad y sin discapacidad.

- Estudio de la efectividad de las medidas de política activa de empleo aplicadas sobre el colectivo.

- Análisis coste - beneficio de las políticas activas de empleo aplicadas sobre el colectivo de las personas con discapacidad.

- Segmentación ocupacional de las personas con discapacidad en el mercado de trabajo español. 


\section{REFERENCIAS BIBLIOGRÁFICAS}

Comisión Europea. (2004). Active labour market programmes for people with disabilities. Facts and figures on use and impact.

Comisión Europea. (2010). Estrategia europea sobre discapacidad 2010 -2020: un compromiso renovado para una Europa sin fronteras.

De Lorenzo, R. (2004). El futuro de los discapacitados en el mundo: el empleo como factor determinante para la inclusión, Revista del Ministerio de Empleo y Seguridad Social no 50, pp $73-89$.

Fundación ONCE, Perspectivas del mundo empresarial respecto a la contratación de personas con discapacidad. Recuperado de: <http://www.redemdisc.es/descargas/Persp empr contr pcd.pdf/>. Disponible en marzo 2017.

Hernández, J. y Millan, J.M. (2015). Las personas con discapacidad en España, inserción laboral y crisis económica, Revista Española de discapacidad, 3 (I), pag 29 -56.

Huete, A. (2012), La discapacidad en las fuentes estadísticas. Examen y propuestas de mejora. Recuperado de: <http://www.cermi.es/esES/Biblioteca/Lists/Publicaciones/Attachments/273/01LA\%20DISCAPACIDAD\%20EN\%20LAS\%20FUENTES.pdf/>. Disponible en enero 2017.

Martín, I. (2016), La discapacidad en las estadísticas del Impuesto sobre la Renta de las Personas Físicas, Ed: Cinca.

Pallisera, M., Fullana, J. y Villa, M. (2005). La inserción laboral de personas con discapacidad. Desarrollo de tres investigaciones acerca de los factores favorecedores de los procesos de inserción, Revista de investigación educativa, Vol. 23, no 2, pag 295 $-313$.

Pereda, C., De Prada, M.A y Actis W. (2003). La inserción laboral de las personas con discapacidades, Colección Estudios Sociales no 14, Obra Social La Caixa.

Pereda, C., De Prada, M.A y Actis W. (2012). Discapacidades e inclusión social, Colección Estudios Sociales no 33, Obra Social La Caixa.

Sanchez, M. y Diaz, M. (2016), Discapacidad vs empleo: Aspectos a considerar para la inclusión en el empleo ordinario, Ed: Dykinson.

Vallas, J., Vila, M. y Pallisera, M. (2004). La inserción de personas con discapacidad en el trabajo ordinario. El papel de la familia, Revista de Educación, no 334, pag 99-117.

VV.AA. (1997), La discapacidad en el siglo XXI. Plan de Acción: Una propuesta de futuro, Ed: Instituto de Migraciones y Servicios Sociales.

VV.AA. (2003), La integración laboral de las personas con discapacidad en la CAPV, Informe extraordinario de la institución del Ararteko al Parlamento Vasco. 